\title{
Valsalva retinopathy in pregnancy: an obstetric dilemma
}

\author{
Vismaya Kaveri ${ }^{1}$, Manjula S. K. ${ }^{2 *}$, Bhargavi Pawar $^{3}$
}

\begin{abstract}
${ }^{1}$ Department of Obstetrics and Gynecology, Yenepoya Medical College, Mangalore, Karnataka, India
${ }^{2}$ Department of Obstetrics and Gynecology, ${ }^{3}$ Department of Ophthalmology, St. John's Medical College, Bangalore, Karnataka, India
\end{abstract}

Received: 04 September 2017

Accepted: 29 September 2017

\section{*Correspondence:}

Dr. Manjula S. K.,

E-mail: thatva@rediffmail.com

Copyright: () the author(s), publisher and licensee Medip Academy. This is an open-access article distributed under the terms of the Creative Commons Attribution Non-Commercial License, which permits unrestricted non-commercial use, distribution, and reproduction in any medium, provided the original work is properly cited.

\begin{abstract}
Valsalva retinopathy is a unilateral or bilateral condition that occurs when increased intra-thoracic or intra-abdominal pressure transmitted to the eye causes a sharp rise in the intraocular venous pressure, and rupture of superficial retinal capillaries. Pregnancy is known to be a risk factor for Valsalva retinopathy. A 29-year-old G2A1 at 38 weeks of gestation previously normotensive presented with unilateral sudden decrease in vision of 1-day duration. Fundoscopy revealed pre-retinal and vitreous hemorrhage of right eye. A diagnosis of Valsalva retinopathy of pregnancy was made after excluding other probable causes for hemorrhage. She had an elective cesarean section with no further deterioration of vision or recurrence of hemorrhage.
\end{abstract}

Keywords: Obstetric dilemma, Retinopathy in pregnancy, Valsalva retinopathy

\section{INTRODUCTION}

The Valsalva's maneuver was named after the Italian anatomist Antonio Maria Valsalva (1666-1723) who defined the valsalva ligaments and anatomy related to the forcible exhalation effort against a closed glottis. ${ }^{1}$ Valsalva retinopathy is a unilateral or bilateral condition that occurs when increased intra-thoracic or intraabdominal pressure transmitted to the eye causes a sharp rise in the intra-ocular venous pressure, and rupture of superficial retinal capillaries. ${ }^{2}$ The patient often gives a history of a recent strenuous physical act, which could have increased the intra-thoracic pressure. Pregnancy is known to be a risk factor for Valsalva retinopathy. ${ }^{3}$

\section{CASE REPORT}

A 29-year-old G2A1 at 38 weeks of gestation presented with sudden decrease in vision of 1-day duration in the right eye to the department of Obstetrics and Gynecology, St. John's Medical College, Bangalore. The decreased vision was painless, non-progressive, not associated with preceding trauma or any ocular symptoms and was noted after straining. Her antenatal period was uneventful. Her past medical history was unremarkable except for bronchial asthma. On examination BP was $140 / 90 \mathrm{mmHg}$ with good fetal wellbeing. Ophthalmic examination showed a visual acuity of $6 / 24$ in the right eye and 6/9 in the left eye.

Intraocular pressure was $16 \mathrm{mmHg}$ in both eyes. Fundus examination of right eye showed pre-retinal and vitreous hemorrhage in the macular region (Figure 1). The examination of the left eye was normal (Figure 2). Complete blood count, coagulation profile, fasting sugar, liver function test done to rule out other causes of hemorrhage including atypical preeclampsia with HELLP syndrome. A clinical diagnosis of Valsalva retinopathy was made, and pregnancy was terminated by elective cesarean section.

During postoperative period patient was observed to have preeclampsia and repeat fundus examination did not show any increase in retinal hemorrhage or any hypertensive changes. Follow up after 2 weeks of 
delivery showed complete resolution of retinal hemorrhage and normal vision.

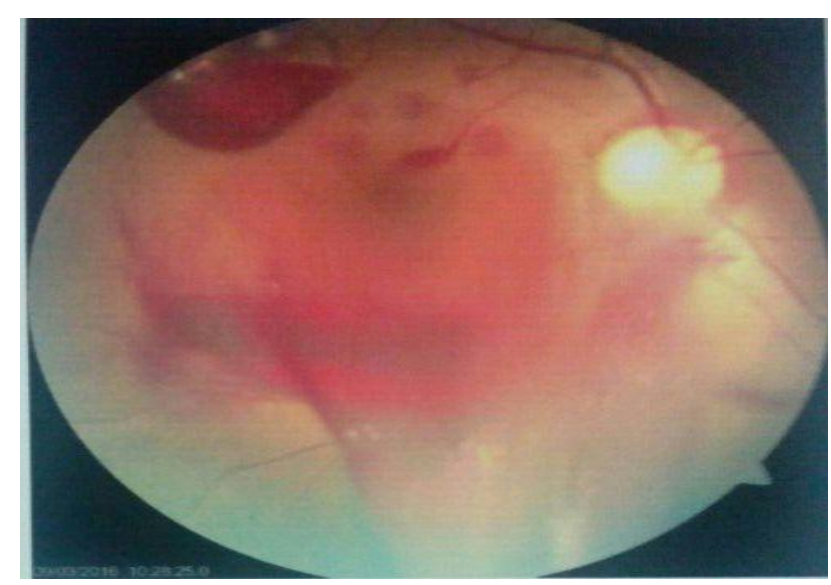

Figure 1: Right eye- Pre-retinal and vitreous hemorrhage in the macular region.

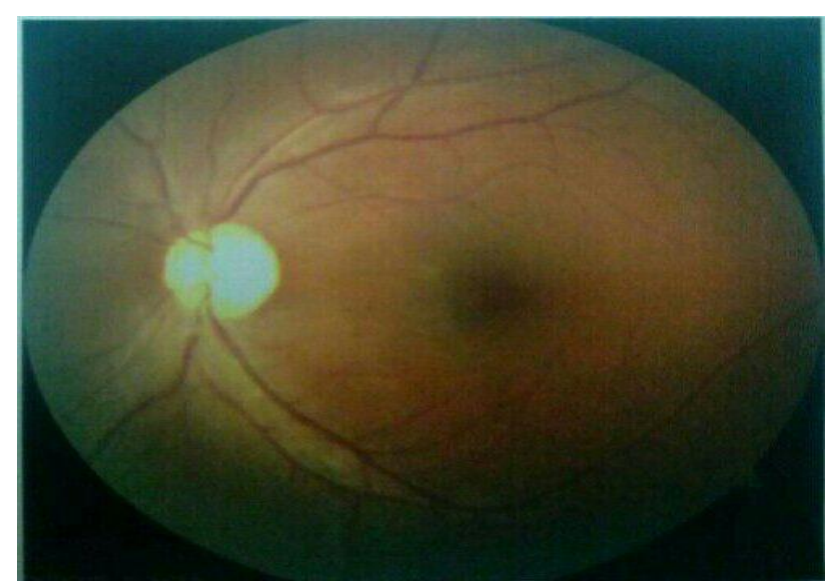

Figure 2: Left eye-normal.

\section{DISCUSSION}

Valsalva retinopathy was first described by Duane in 1972 for preretinal hemorrhages occurring as a result of sudden rise in venous pressure as seen in Valsalva manoeuvre. ${ }^{4}$ It is an uncommon condition and has been reported to occur with various forms of Valsalva stress such as weight lifting or heavy lifting, constipation, vomiting, coughing, sneezing, vaginal delivery (labor), vigorous sexual activity, vigorous dancing, bungee jumping and continuous positive pressure ventilation., ${ }^{4,5}$ The sudden increase in the central venous pressure due to Valsalva stress is transmitted right up to the perifoveal capillaries which may rupture leading to pre-retinal hemorrhage at macula ${ }^{6}$

Pregnancy exerts metabolic, immunological, hormonal and hematological changes with thrombocytopenia in the mother that represent risk factors for Valsalva retinopathy. ${ }^{7}$ Pregnancy is identified as a predisposing factor for Valsalva retinopathy. Diagnosis is made by exclusion after ruling out other probable causes for retinal hemorrhage such as hypertension, diabetes, blood dyscrasias, sickle cell disease, anemia and previous ocular vein occlusion.

Elevation of intra-abdominal pressure during pregnancy increases the baseline intravenous pressure which may be increased further during labor, predisposing to retinal hemorrhages following a Valsalva maneuver. Whether vaginal delivery poses a risk of recurrence or exacerbation of hemorrhage is not known as there are no reports documenting recurrence. There have been cases reported with Valsalva retinopathy following anesthesia for Caesarean section. ${ }^{8}$ Both regional and general anesthesia trigger an increase in venous pressure and the latter may induce Valsalva maneuver during extubation. Hence, the mode of delivery in patients with Valsalva retinopathy is still a dilemma.

Valsalva maneuver typically results in sub-internal limiting membrane hemorrhages with a predilection for macula, but sub-retinal or intravitreal hemorrhages can occur. The prognosis of this condition is good with spontaneous resolution in most patients over a few months. Some patients may have a poor visual outcome, which has been attributed to retinal pigmentary changes at the macula. ${ }^{7}$

Different techniques have been employed to treat premacular hemorrhage. Nd-YAG or early YAG laser to allow drainage of hemorrhage into vitreous cavity which allows restoration of vision. During pregnancy Valsalva retinopathy is managed conservatively as current literature lacks data to address the safety of YAG laser or surgical intervention.

\section{CONCLUSION}

Valsalva retinopathy may be precipitated by a number of diverse causes, including pregnancy. Spontaneous resolution with recovery of normal vision is the rule in majority of cases. Valsalva retinopathy at term gestation may be managed by cesarean section to prevent a rebleed that may be precipitated by a vaginal delivery. If it occurs in the second or early third trimester and resolves spontaneously a trial of labor can be allowed since recurrences are uncommon.

\section{Funding: No funding sources \\ Conflict of interest: None declared \\ Ethical approval: Not required}

\section{REFERENCES}

1. Jones WL. Valsalva maneuver induced vitreous hemorrhage. J Am Optom Assoc. 1995;66(5):301-4.

2. Chapman-Davies A, Lazarevic A. Valsalva maculopathy. Clin Exp Optom. 2002;85:42-45.

3. Deane JS, Ziakas N. Valsalva retinopathy in pregnancy. Eye 1997;11:137. 
4. Duane TD. Valsalva haemorrhagic retinopathy. Trans Am Ophthalmol Soc. 1972;70:298e313.

5. Cheung CM, Durrani OM, Kirkby GR. Sudden visual loss in asthma. J R Soc Med. 2002;95(7):359.

6. Col Shrikant Waikar, Brig V.K. Srivastava. Valsalva retinopathy in a young healthy individual. Med J Armed Forces India. 2012;69:193-5.

7. Al-Mujaini AS, Montana CC. Valsalva retinopathy in pregnancy. J Med Case Reports. 2008;2:10.
8. El-Defrawy H, Sallam A, McKechnie CJ, Zambarakji H. Valsalva retinopathy in pregnancy. Should we treat? JRSM short reports. 2011 Jun;2(6):1-3.

Cite this article as: Kaveri V, Manjula SK, Pawar B. Valsalva retinopathy in pregnancy: an obstetric dilemma. Int J Reprod Contracept Obstet Gynecol 2017;6:5170-2. 